\title{
Predictability and unpredictability in optical system optimization
}

\author{
Maarten van Turnhout ${ }^{*}$ and Florian Bociort \\ Optics Research Group, Delft University of Technology \\ Lorentzweg 1, 2628 CJ Delft, The Netherlands
}

\begin{abstract}
Local optimization algorithms, when they are optimized only for speed, have in certain situations an unpredictable behavior: starting points very close to each other lead after optimization to different minima. In these cases, the sets of points, which, when chosen as starting points for local optimization, lead to the same minimum (the so-called basins of attraction), have a fractal-like shape. Before it finally converges to a local minimum, optimization started in a fractal region first displays chaotic transients. The sensitivity to changes in the initial conditions that leads to fractal basin borders is caused by the discontinuous evolution path (i.e. the jumps) of local optimization algorithms such as the damped-least-squares method with insufficient damping. At the cost of some speed, the fractal character of the regions can be made to vanish, and the downward paths become more predictable. The borders of the basins depend on the implementation details of the local optimization algorithm, but the saddle points in the merit function landscape always remain on these borders.
\end{abstract}

Keywords: optimization, optical system design, saddle points, fractals, basins of attraction

\section{INTRODUCTION}

In optical system design, there are typically many local minima in the merit function landscape, and the solution that will be obtained by local optimization is critically dependent on the choice of the initial configuration. Mathematically speaking, to understand when optimization leads to distinct local minima, the most fundamental way is to look at the socalled basins of attraction. The basin of attraction of a local minimum is the set of initial conditions which lead, after optimization, to that minimum (see Fig. 1).

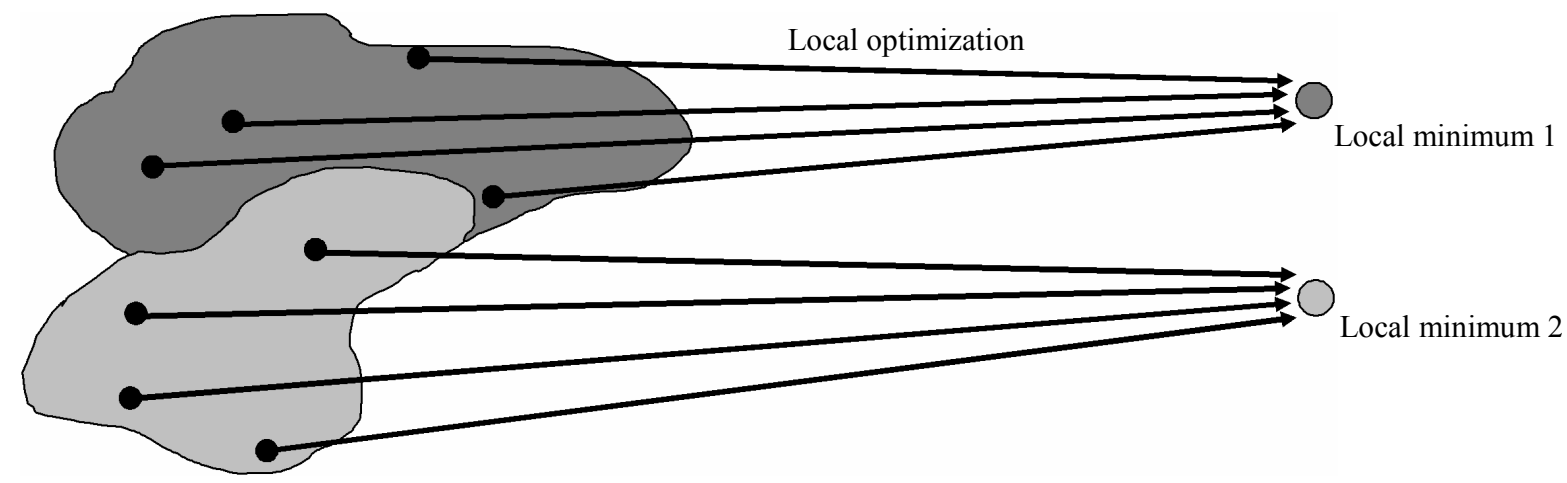

Fig. 1. Illustration of two neighboring basins of attraction. After local optimization, all points in one basin lead to the same minimum.

It is well-known that a change in the optimization strategy or in the settings of the local optimization algorithm can lead to a different result, even when starting from the same configuration ${ }^{1}$. Therefore, it is not surprising that the basins of

*m.vanturnhout@tudelft.nl; phone: +31 15278 8109; fax +31 15278 8105;

Current Developments in Lens Design and Optical Engineering VIII, edited by Pantazis Zakos Mouroulis,

Warren J. Smith, R. Barry Johnson, Proc. of SPIE Vol. 6667, 666709, (2007)

0277-786X/07/\$18 - doi: $10.1117 / 12.734210$

Proc. of SPIE Vol. 6667 666709-1 
attraction change as well. We will illustrate this property by using different methods to obtain the basins of attraction of the same optimization problem. Since computing the basins of attraction is very time-consuming, we will use the simplest example that illustrates our findings: a monochromatic doublet, with only two variables (Sec. 2).

In this paper, the emphasis is on understanding the relationships between basins of attraction and the optimization algorithm that is used or its implementation details. In order to have maximum predictability, we will first use a differential equation to go down the gradient of the merit function. This is basically a steepest descent method with an infinitesimal step size. In this way, we obtain a reference for the basins of attraction obtained with different programs using a damped least-squares algorithm, which are presented in Sec. 3.

In Sec. 3, we will show that for some settings of the local optimization algorithm, the basins of attraction of the doublet example have surprising shapes. With insufficient damping, the sharp borders of the basins are replaced by fractal-like regions. These complicated borders make the local optimization unpredictable. For example, starting points arbitrarily close to each other in a fractal region can lead to different results after we optimize them locally. However, for some applications it can be important to have more predictable optimization paths, i.e. starting points close to each other should typically lead after optimization to the same minimum. We will examine the basins of attraction for three different optimization algorithms, which are based on the damped least-squares method. With sufficient damping, we will see that the borders are regular and the optimization becomes predictable.

\section{METHOD FOR MAXIMUM PREDICTABILITY}

In this paper, we consider a monochromatic doublet (F number 3, field of 3 degrees, wavelength of $587.6 \mathrm{~nm}$, glasses are BSM24 and SF1, and object is at infinity), with curvature $c_{2}$ of the second surface and curvature $c_{3}$ of the third surface as variables, the first curvature is kept constant at $0.019893 \mathrm{~mm}^{-1}$, and the last curvature is used to keep the effective focal length constant at $100 \mathrm{~mm}$. When we optimize the two curvatures, depending on the merit function used, we obtain four or five different local minima, which are shown in Fig. 2. Minima A-D are stable local minima, which are obtained in all runs, but have rather high merit function values, especially minimum D. Minimum E (which also suffers from edge thickness violations), is for our settings less stable, and appears or disappears when we change the software used.

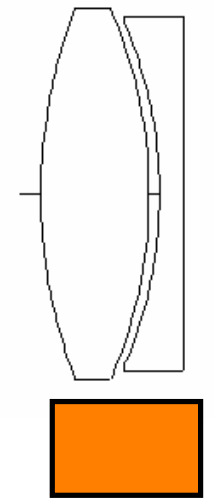

A

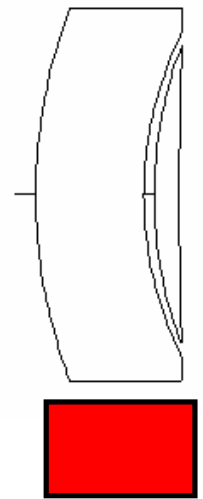

B

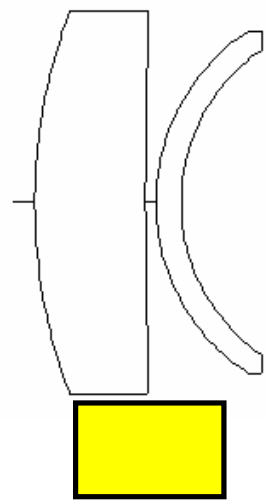

C

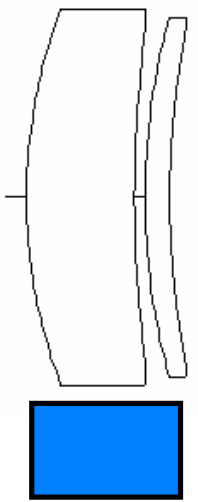

D

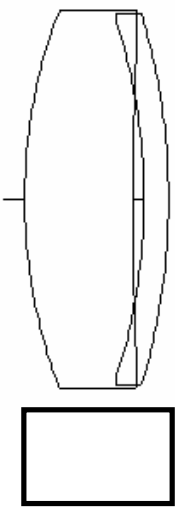

$\mathbf{E}$

Fig. 2. Five monochromatic doublet minima. The corresponding colored boxes under the minima are used when we show the basins of attraction for those minima.

In Fig. 3, we show the equimagnitude contours of the default merit function of CODE V (i.e. the contours along which the merit function remains constant), which is based on transverse aberrations, together with the positions of the minima A-D in the two-dimensional merit function landscape. In all figures, curvature $\mathrm{c}_{2}$ is plotted along the vertical axis, and curvature $c_{3}$ is plotted along the horizontal axis. For the settings of this CODE V run, minimum E did not exist. (It can appear in CODE $\mathrm{V}$ as well with slightly modified settings.) 
We now want to find the shapes of the basins of attraction. In order to have maximum predictability of the downward path from the starting point to the local minimum, we first use a differential equation to follow continuously the gradient of the merit function (MF) downwards:

$$
\frac{d \mathbf{x}}{d s}=-\frac{\nabla \mathrm{MF}}{\|\nabla \mathrm{MF}\|},
$$

where the vector $\mathbf{x}=\left(\mathrm{c}_{2}, \mathrm{c}_{3}\right)$ describes a point in the two-dimensional solution space, and $d s=\sqrt{\|d \mathbf{x}\|^{2}}$ is the arc length along the curved continuous path. We have implemented Eq. (1) in the macro language of CODE V, and we have used a fourth-order Runge-Kutta method with adaptive step size to solve this differential equation. Since solving this differential equation is very time-consuming, we only use Eq. (1) in a first stage of the optimization process. (We use a maximal number of steps.) In a second stage, we continue with the default optimization routine of CODE V. In most cases, solving the differential equation "moves" the starting point for the default optimization routine closer to one of the local minima, where the damped least-squares method behaves adequately.

For computing the basins of attraction for the minima shown in Figs. 2 and 3, we use a grid of equally spaced starting points in the plane $\left(\mathrm{c}_{2}, \mathrm{c}_{3}\right)$. At every grid point, we start with following the gradient downwards by solving Eq. (1). Then, when the maximal number of steps is reached, we continue the process by optimizing the point with the default optimization of CODE V. Depending on which local minimum we obtain, we color the starting point with the corresponding color for that minimum (see Fig. 2). Figure 4 shows the basins of attraction for a grid of $101 \times 101$ points. It is important to note that the three saddle points in the merit function landscape (green dots at the crossing of two segments of the equimagnitude merit function contours) are situated exactly on the borders.

In this case, the borders of the basins (which will be the reference for the following runs) are smooth curves and the basins are compact regions in the merit function space. However, there are some minor artifacts in Fig. 4, especially close to the upper and lower part of the border between the basins of minimum A and D. By continuing with the differential equation further, these artifacts disappear (with a considerable increase in computation time).

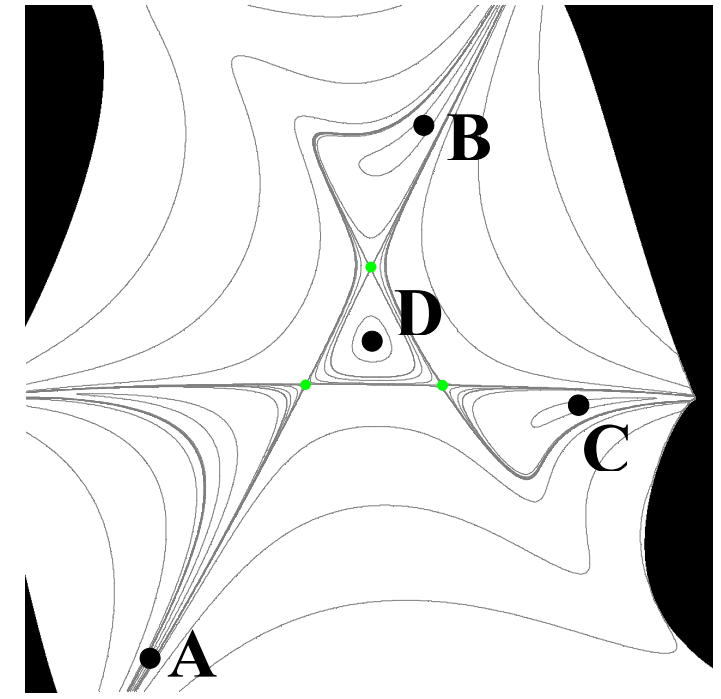

Fig. 3. Merit function equimagnitude contours of the twodimensional monochromatic doublet. The larger points A, B, $\mathrm{C}$, and D, correspond to the local minima shown in Fig. 2. The smaller points, situated there where equimagnitude contours cross, are saddle points. In all figures of the same kind, the compact black regions contain configurations that suffer from ray failure (a ray misses a surface or total internal reflection).

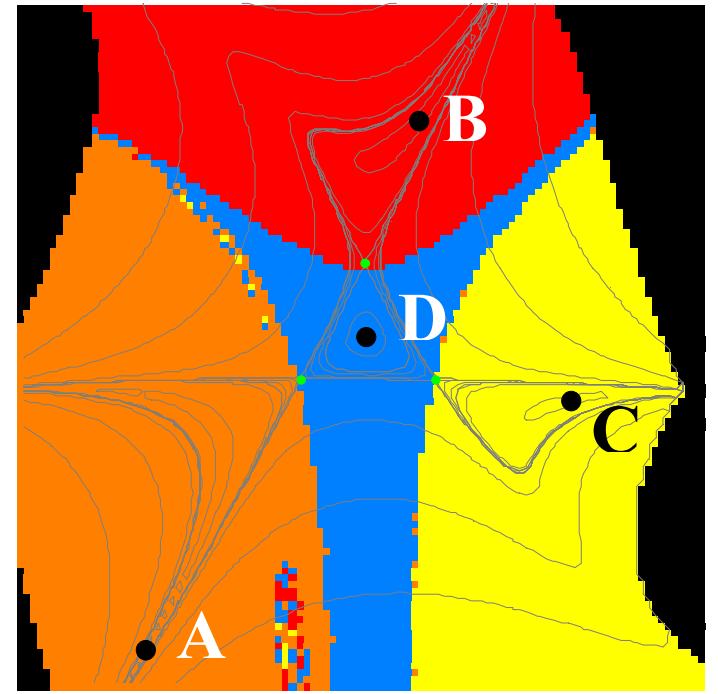

Fig. 4. Basins of attraction of the two-dimensional monochromatic doublet (on a grid of $101 \times 101$ points) obtained by using a differential equation in a first stage of the optimization process in CODE V. (The gray contours are the equimagnitude contours of the default merit function.) The colors correspond to the local minima as shown in Fig. 2. 


\section{BASINS OF ATTRACTION OBTAINED WITH DAMPED LEAST-SQUARES ALGORITHMS}

In this section, we show how the basins of attraction of the doublet minima change if we change the optimization strategy or some settings in the local optimization algorithm. We use the optimization algorithms of three different programs to generate the basins and compare the results. We use a home-made code (OptSys, author Joseph Braat), and two commercial codes (CODE V and ZEMAX), all three using the damped least-squares method.

The optimization algorithm of OptSys is based on the Levenberg-Marquardt damped least-squares method, with an adaptive damping factor. The initial damping in this program can be controlled by the user. It turns out that the value of the initial damping determines the form of the basins. When the initial damping is sufficiently large, we obtain the basins shown in Fig. 5a. It is known that with high damping the behavior of the damped least-squares optimization begins to resemble the behavior of the steepest descent method ${ }^{1}$. Therefore, it is not surprising that the basins borders in Fig. 5a are qualitatively of the same kind as the ones shown in Fig. 4. (Recall that the method used in Fig. 4 is essentially a steepest descent with infinitesimal step size.) However, with OptSys we obtain an extra basin (for minimum E). In this code, when ray failure occurs during optimization, the algorithm stops the optimization process. (CODE V and ZEMAX can seemingly escape from these situations.) The corresponding starting points are then drawn in black.

In Figs. 5b-c, we decreased the damping factor, with a minimum of damping in Fig. 5c. By decreasing the damping, the regular basin shapes change into more complicated ones with increasingly diffuse borders. There also appear regions of poor convergence (shown in purple). Since less damping allows the algorithm to take bigger steps in the merit function landscape, the number of ray failures increases. Therefore, there are more black regions in Fig. 5c than in Figs. 5a-b.

We also examined these basin borders with commercial optical design software. We used CODE V and ZEMAX with their default optimization algorithms and a merit function based on transverse aberrations. In CODE V, for each initial set of curvatures, the system was iterated with a maximum of 100 optimization cycles (ZEMAX with 30 cycles). For the settings we use here, minimum E (see Fig. 2) does not exist for the merit function of CODE V and ZEMAX.

In Fig. 6, we show the basins of attraction obtained with CODE V on a grid of 1001x1001 points (a-c), and ZEMAX on a grid of $401 \times 401$ points (d). As in the previous figures, starting points for which the initial configuration suffers from ray failure are in the compact regions at the periphery (black). Note the very complex shapes and the very fine-scale structure in the basins of attraction, which is present on all scales, as shown in Figs. 6a-c. On a small scale, the basins appear to be finely interwoven, indicating the fractal nature of the basin boundaries. These complicated border structures are related to unpredictable results in the optimization process. When we compare Fig. 6a with Fig. 5c, we observe certain similarities. However, since CODE V can handle ray failure occurring during optimization better than OptSys, Fig. 6a has less black regions than Fig. 5c.

To understand why the basin borders are so complicated, we examine the sequence of steps in the two-dimensional variable space that are taken by the optimization routines of CODE V and ZEMAX. Examples of optimization paths are shown in Fig. 7 (on a grid of $401 \times 401$ points). The optimization starts from points in the parameter space, indicated with "START", and the result after each optimization cycle is shown in the figure by black dots. The black dots are connected with black lines, showing the direction of the optimization paths in the merit function landscape.

In Fig. 7, we see an interesting behavior of optimizations started in a fractal region. Surprisingly, the four separate optimizations in Fig. 7a converge to four different local minima. Before the optimization finally converges to a local minimum, optimization started in a fractal region first displays an unpredictable chaotic path in the merit function landscape. Note that the starting points in Fig. 7 a are very close to each other, but during optimization, the distances between the points increase. After a number of iterations, the optimization starts to converge towards one of the four local minima. This type of behavior is known as a chaotic transient ${ }^{2}$. It is known that with low damping the behavior of the damped least-squares optimization begins to resemble the behavior of quasi-Newton methods ${ }^{1}$, and examples are known where quasi-Newton methods produce fractal-like basins ${ }^{3,4}$. 


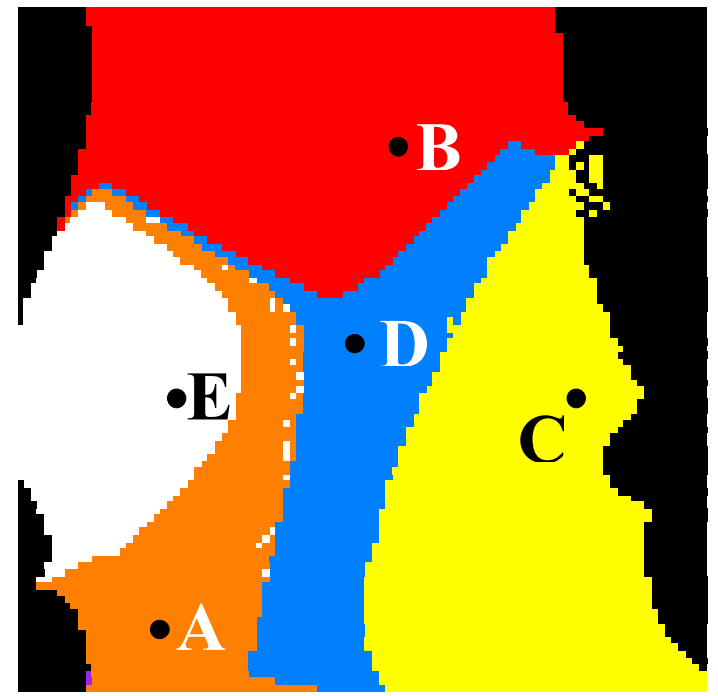

(a)

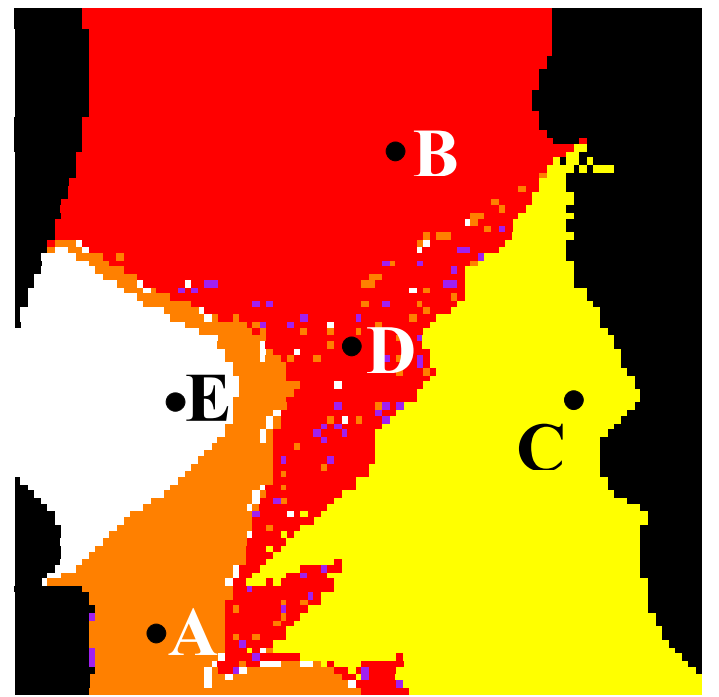

(b)

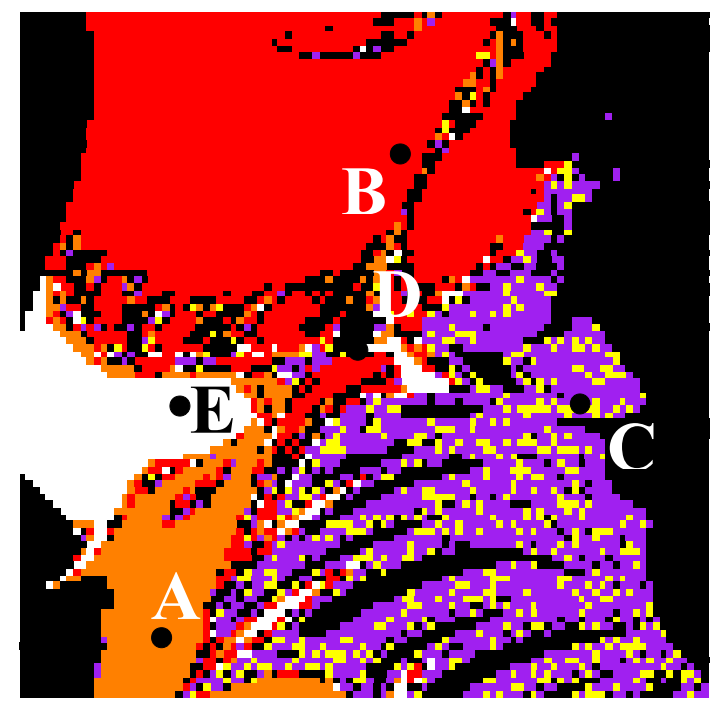

(c)

Fig. 5. Basins of attraction of the two-dimensional monochromatic doublet, obtained with OptSys, for different values of the initial damping factor on a grid of $101 \times 101$ points. Without sufficient damping, the optimization algorithm can easily jump over the flat central area. For example, when the initial damping factor in OptSys is too low, minimum D is not found anymore. a) Large damping, b) intermediate damping, and c) low damping. The colors correspond to the local minima as shown in Fig. 2. The purple points in b) and c) indicate configurations that have poor convergence.

The irregular shape of the paths in Fig. 7a and the similarity between Figs. 5c and 6a suggest that in Fig. 6a, the optimization algorithm is using low damping. The results shown in Fig. 5 suggest that in order to make the borders more regular, a higher damping factor in the optimization algorithm is needed. However, in the present versions of CODE V and ZEMAX, the user does not have the possibility to influence the automatically computed damping factor. For preventing the optimization algorithm to jump unpredictably, we have implemented an external damping factor in the macro language of CODE V. Our external damping factor does not change the direction of the optimization step, but it decreases the step size. The direction of the variable change at each iteration is still determined by the optimization algorithm. Our procedure is illustrated in Fig. 8. Note that this technique is not equivalent to a change of the automatic 
internal damping of the optimization algorithm, but is, as shown below, only a partially satisfactory solution. (For a discussion of a typical way to implement internal damping, see for example Ref. 1.)

Fig. 9 shows the basins of attraction of the doublet in CODE V with high external damping. In comparison with Fig. 6a, the borders in Fig. 9 are regular and the basins are compact, which confirms our hypothesis that in Fig. 7a the chaotic transients are caused by a damping that is too low for producing a predictable result. However, unlike the internal damping in Fig. 5, controlling external damping does not lead to basin shapes close to the reference ones in Fig. 4.

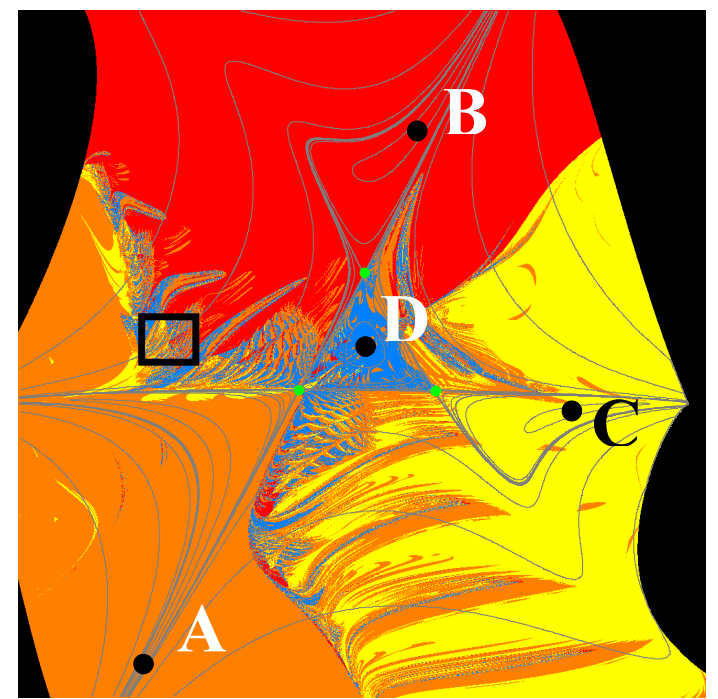

(a)

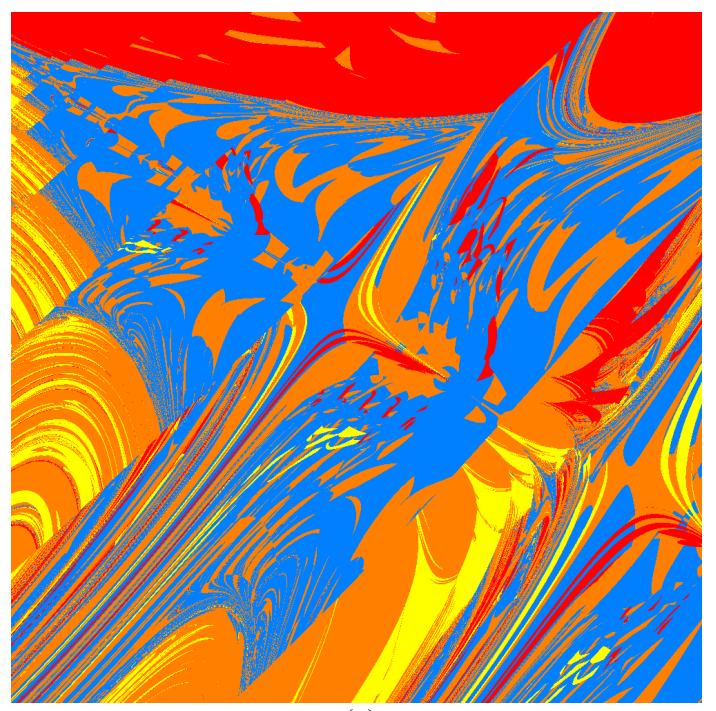

(c)

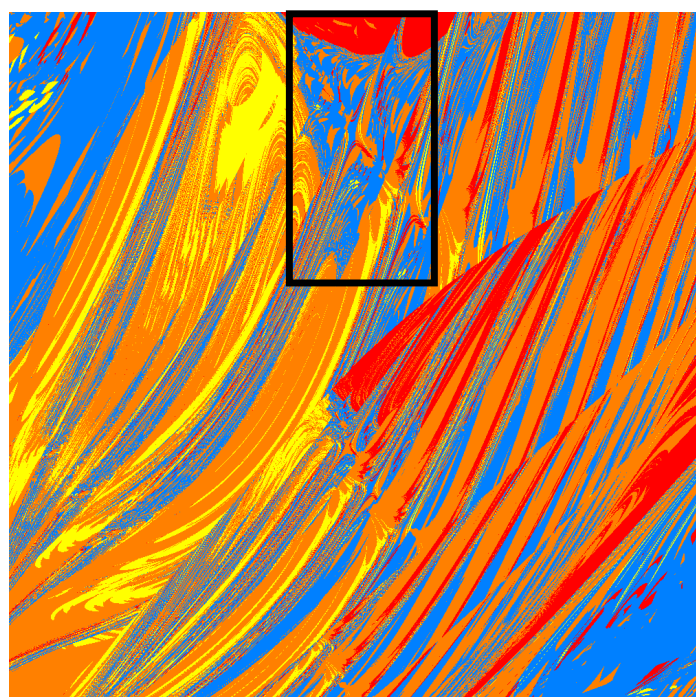

(b)

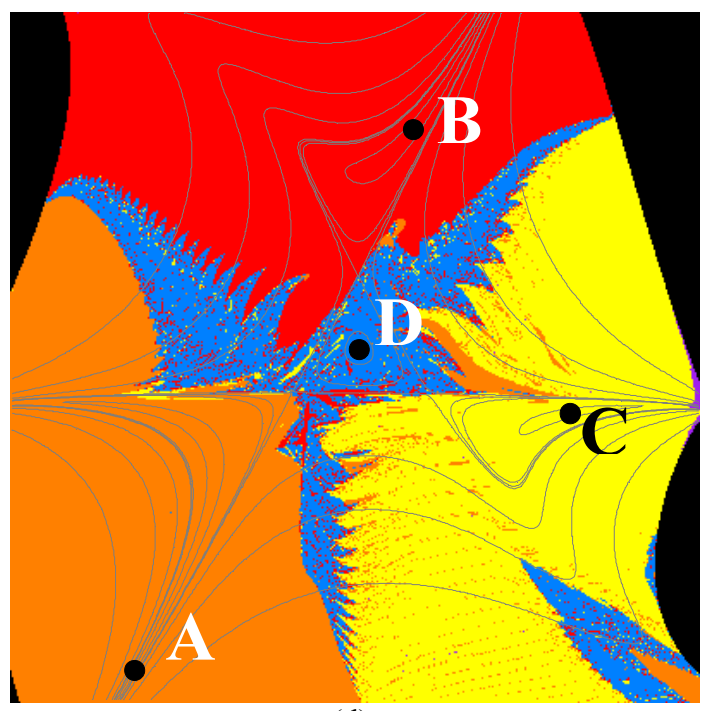

(d)

Fig. 6. Basins of attraction of the two-dimensional monochromatic doublet obtained with commercial software. (The gray contours are the equimagnitude contours of the merit function.) a) Default optimization of CODE V, b) magnification of the small black rectangle in figure (a), c) magnification of the small black rectangle in figure (b), d) damped least-squares optimization of ZEMAX. Note some sharp spikes on the basin borders similar with those in Fig. $5 \mathrm{~b}$. 


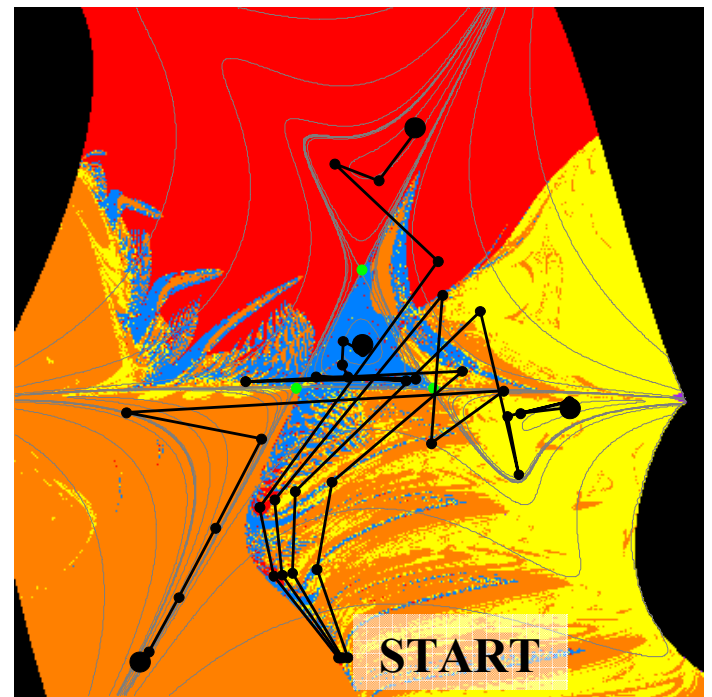

(a)

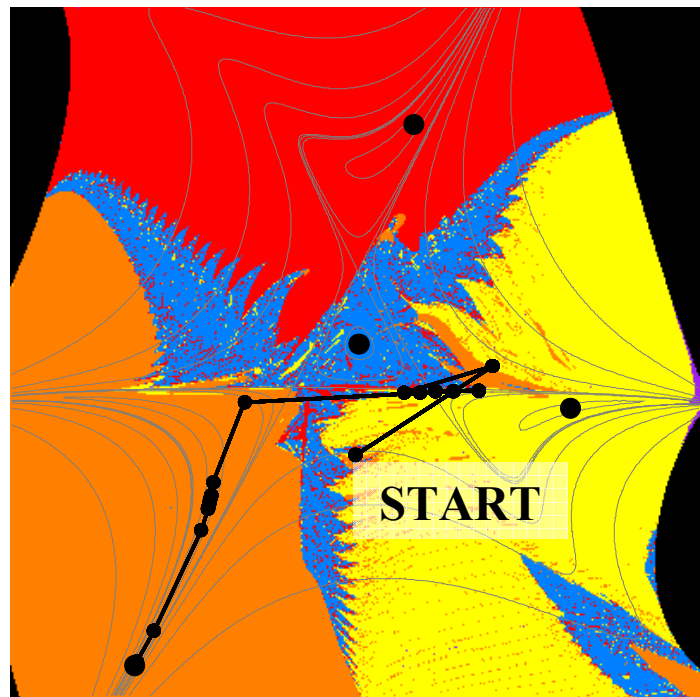

(b)

Fig.7. Examples of optimization paths (black lines) in the variable space of the doublet. The starting points are at "START". Note that the sequence of iterations will always remain inside the same basin as for the starting point, independent of the basin shape. a) Optimization paths of four starting points obtained with the default optimization of CODE V. b) Optimization path of a starting point obtained with the damped least-squares optimization of ZEMAX.

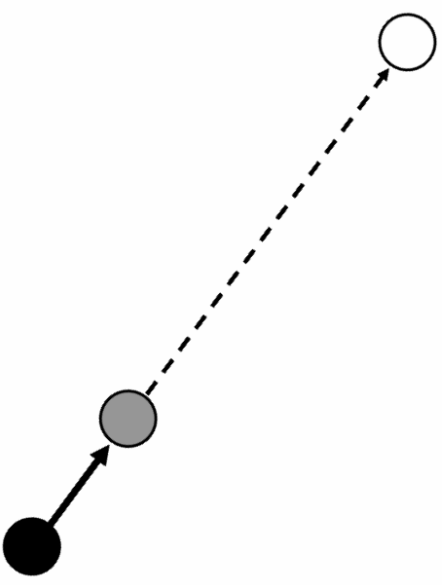

Fig. 8. Illustration of our external damping factor. The starting point is shown in black, and the CODE V result after one optimization step is shown in white. The external damping factor damps the optimization in such a way that it does not change the direction, but only shortens the step size. Our result after external damping is shown in gray.

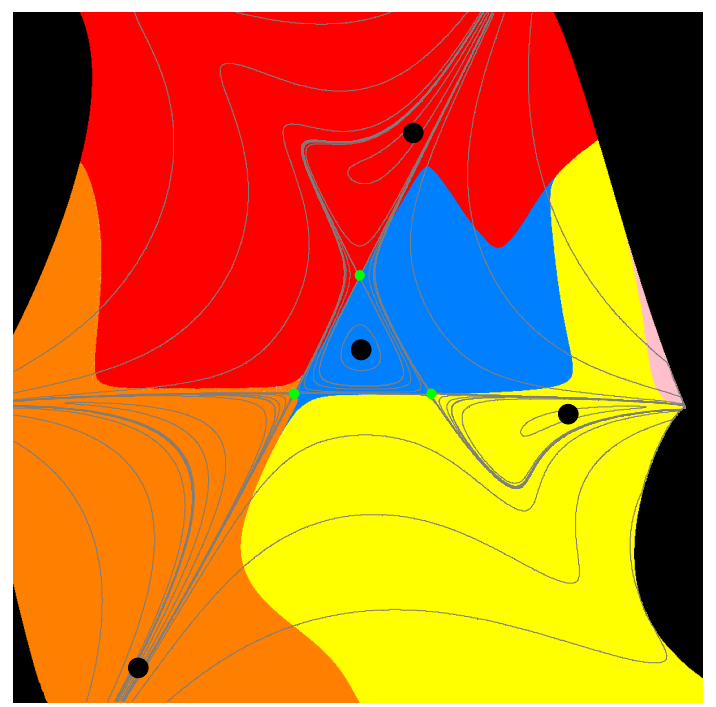

Fig. 9. Basins of attraction made with the default optimization of CODE $\mathrm{V}$ with high external damping (see text) on a grid of $1001 \times 1001$ points.

By comparing Figs. 4, 6, and 9, we observe an interesting property of the saddle points in the merit function landscape. The border shapes vary considerably, but we can find points that always remain points on the basin borders, whether they are regular curves or have fractal shapes. Such border points, which are unaffected by the choice of algorithm or damping, are the saddle points. Therefore, if we can find a saddle point, we can use it to move from one basin to a neighboring one in a more systematic way. In our research at TU Delft, saddle points in the optical merit function landscape play a central role ${ }^{5-9}$. 
Finally, we want to emphasize that the chaotic transients observed in our doublet example are not typical for the optimization behavior in usual design problems with software that computes the damping automatically, without any user intervention. Rather, despite its simplicity, we have in our doublet example many (four and sometimes even five) minima, all with high merit function values in a landscape with only two dimensions. In such situations, chaotic transients appear more easily. Our preliminary studies with more complex systems suggest that for deeper minima the behavior is more regular, especially for starting points that are close enough to the minima. However, far away from the local minima, low damping values that are ideal to increase computational speed, are not necessarily optimal when more predictability of the final result is required. In Fig. 5a, stronger initial damping brings the borders closer to the ideal shape (Fig. 4), and then, unless they are on opposite sides of the border, starting points close to each other typically converge to the same minimum.

\section{CONCLUSIONS}

To understand when the optimization goes to a certain local minimum or to a neighboring one, the most fundamental way is to look at the basin of attraction of that minimum. The basin borders depend on the implementation details of the used algorithm and on numerical parameters, such as the damping factor. However, the saddle points in the merit function landscape are always on the border.

We show that, in certain situations, local optimization methods which are optimized for speed can have an unpredictable behavior. Then, the borders of the basins of attraction have a fractal-like shape. In fractal regions, starting points very close to each other lead after optimization to different local minima. Before it finally converges to a local minimum, optimization started in a fractal region first displays chaotic transients. When the local minima are sufficiently deep, typically we encounter a more predictable optimization behavior.

In order to have maximum predictability, we use as reference a differential equation to follow continuously the gradient of the merit function downwards. In this way, or with damped least-squares methods with sufficient damping, we can obtain smooth basin borders even in optimization problems that have a natural propensity for fractal basins, such as our doublet. Therefore, the main cause for unpredictability is to be found in the optimization algorithm. Strategies for choosing the damping factor, which turn out to be optimal close to local minima, are not necessary optimal far away from local minima or near the borders of the basins. This paper shows that choosing an optimal automatic damping is perhaps more complicated than previously assumed. For some applications, a user-defined trade-off between optimization speed (lower damping) and predictability (higher damping) might be desirable.

We would like to thank Joseph Braat and Leo Beckmann for stimulating discussions, and we acknowledge the support of the Dutch Technology Foundation STW. We also acknowledge the use of an educational license of CODE V.

\section{REFERENCES}

1. H. Gross et al., Handbook of Optical Systems, Volume 3, Chapter 32, Wiley-VCH, Weinheim, 2007.

2. E. Ott, Chaos in Dynamical Systems, 2nd Edition, Chapter 5, p.168, Cambridge University Press, Cambridge, 2002.

3. D.G. Mayer et al., Efficiency and Fractal Behaviour in Optimisation Methods on Multiple-Optima Surfaces, Agricultural Systems 36, p. 315-328 (1991).

4. Y.Z. Xu et al., Using Fractals to Solve the Multiple Minima Problem in Molecular Mechanics Calculation, Journal of Computational Chemistry, Vol. 21, p. 1101-1108 (2000).

5. F. Bociort and M. van Turnhout, Generating saddle points in the merit function landscape of optical systems, Proc. SPIE 5962, 0S1-8 (2005).

6. O. Marinescu and F. Bociort, Saddle points in the merit function landscape of lithographic objectives, Proc. SPIE 5962, 0T1-8 (2005).

7. O. Marinescu and F. Bociort, Designing lithographic objectives by constructing saddle points, Proc. SPIE 6342, 6342L (2006).

8. F. Bociort and M. van Turnhout, Looking for order in the optical design landscape, Proc. SPIE 6288, 628806, (2006).

9. http://www.optica.tn.tudelft.nl/users/bociort/SPC_guide.zip 